\title{
26 \\ PLATFORMS FOR SCALABLE FLEXIBLE AUTOMATION CONSIDERING THE CONCEPTS OF IEC 61499
}

\author{
Werner E. Rumpl ${ }^{1}$, Franz Auinger ${ }^{1}$, Christoph Dutzler ${ }^{2}$, Alois Zoitl ${ }^{2}$ \\ ${ }^{1}$ Profactor GmbH, Wehrgrabengasse 1-5, A-4400 Steyr, Austria, werner.rumpl@profactor.at \\ ${ }_{2}^{2}$ Institut für Automatiseriungs- und Regelungstechnik, Gusshausstr. 27-29, A-1040 Wien, \\ Austria,cd@infa.tuwien.ac.at
}

\begin{abstract}
This work introduces approaches and embedded execution platforms for implementing the IEC 61499 (IEC, 2000) execution model. The IEC 61499 execution model is described as defined in the IEC 61499 standard. Issues that currently are not in the scope of the standard are discussed and by means of an illustrated IEC 61499 example application, it introduces different approaches that are beyond the standards' definition for embedding IEC 61499 on execution platforms with restricted memory, performance and operating system. Moreover the execution platforms as well as their implemented execution model are described and their advantages and disadvantages are illustrated.
\end{abstract}

\section{INTRODUCTION}

Currently industrial automation is divided into at least two main traditional domains: programmable logical controllers (PLC) and distributed control systems (DCS). Due to the trend of industrial automation equipping even small field devices with intelligent controllers in order to enable performing tasks autonomously, the concepts of PLCs and DCSs are merged and extended to distributed industrial process, measurement and control systems (IPMCSs). An IPMCS forms one base of Scalable Flexible Automation (SFA) (Christensen, 2002) and consists of a network of "smart" and heterogeneous field devices (different vendors, different applications) interacting with each other via standardized (field-) bus- connections. The IEC 61499 standard has been developed to enable and to ease engineering of IPMCSs by meeting the fundamental requirements for SFA such as reusability, configurability, portability of applications, scalability, flexibility, interoperability and reconfiguration, the last-mentioned especially for Holonic Manufacturing Systems (Christensen, 2002)(Holonic, 1998). As defined in IEC 61499 standard part 1 (IEC, 2000) the execution of IEC 61499 Function Blocks (FBs) is based on an event triggered methodology. However, currently the standard describes the execution of one IEC 61499 Function Block (FB). Considering a network of interacting FBs, also called an IEC 61499 application, there is the need to extend the IEC 61499 execution model by considering losses of overloaded events and its associated data that mav cause unpredictable applications. 


\subsection{Motivation}

Up to now there were only few trials to implement the execution model of the IEC 61499. Published approaches are based on PC based IEC 61499 runtimes using high performance processors (e.g.: Pentium or more) with sufficient memory and powerful operating systems (Holobloc, 2002). By considering embedded platforms with slower processors, less memory and without any powerful operating system or even no one, there is the need for alternative execution models in order to verify and validate the IEC 61499 execution model by means of realistic small and "smart" field devices that are realized with embedded systems.

\section{THE IEC 61499 EXECUTION MODEL}

\subsection{Overview}

The IEC 61499 standard describes architectures and models for distributed systems and is not primary considered being a programming methodology (Lewis, 2001). The IEC 61499 provides a set of models for the use of FBs in IPMCSs. These FBs encapsulate user-defined algorithms to perform different tasks (e.g.: measurement of temperature, control of a conveyor belt). The invocation of the algorithms processing data is triggered by events. Therefore one IEC 61499 FB consists of two main layers - one event layer and one data layer (see Figure 1). IEC 61499 describes different models of FBs, namely Basic Function Blocks, Composite Function Blocks, Service Interface Function Blocks, Adapters and a Subapplications Model. Here we focus on the relevant ones to provide a common understanding that is necessary for this work.

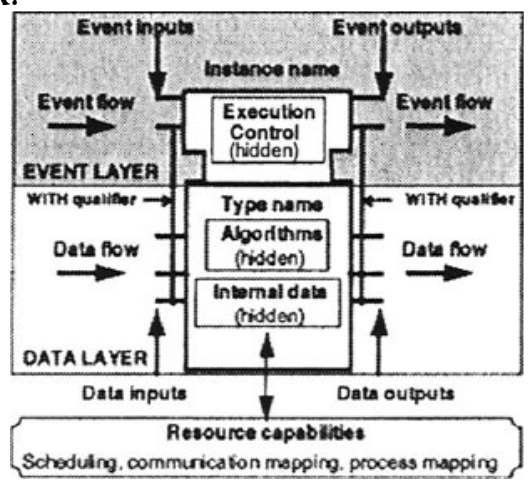

Figure 1 - Basic Function Block

As shown in Figure 1, each FB can be built up with zero or more event in- and outputs as well as with zero or more data in- and outputs. To get a network of interacting FBs, event/data outputs of one FB can be connected to event/data inputs of other FBs or even the same one - for detailed connection rules see (IEC, 2000). This network of FBs forms an IEC 61499 application that can be distributed among different Devices and Resources. One IEC 61499 system (equivalent with one IPMCS) consists of one ore more devices, resources and applications (see Figure 2) (IEC, 2000). 


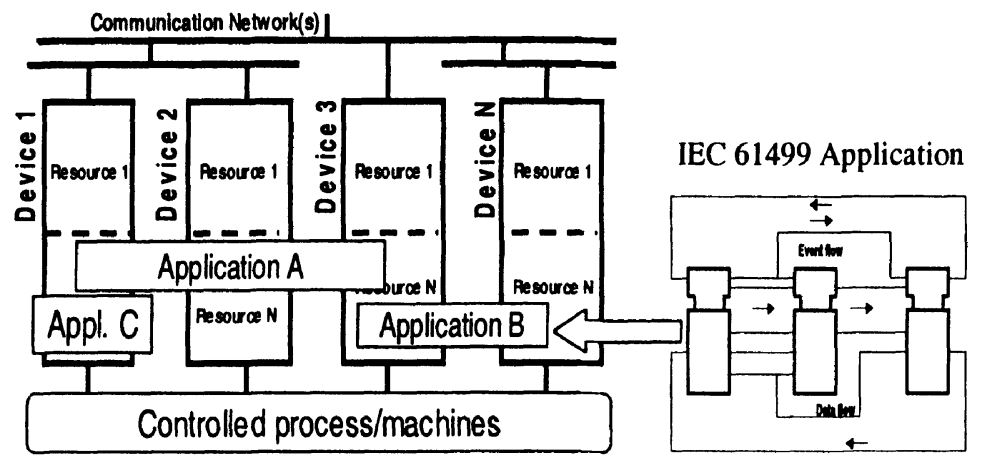

Figure 2 - Overview of an IEC 61499 system

\subsection{The IEC 61499 Execution Model According to the Standard}

As mentioned in chapter 2.1 IEC 61499 FBs are executed on the behalf of events. If an input event occurs, the algorithms may process input data, may update output data and may generate one or more event outputs. A so-called WITH qualifier is to determine, which data in- or output(s) to sample when its associated in- or output event(s) occurs - e.g.: REQ WITH SD, TEMP; means that the input variables SD and TEMP will be sampled if the event input REQ is invoked. The WITH qualifier is graphically represented by a line (see Figure 1). The IEC 61499 Standard Part 1 defines 8 times to illustrate the execution of one Basic FB with a single event input, a single algorithm and a single event output (see Figure 3).

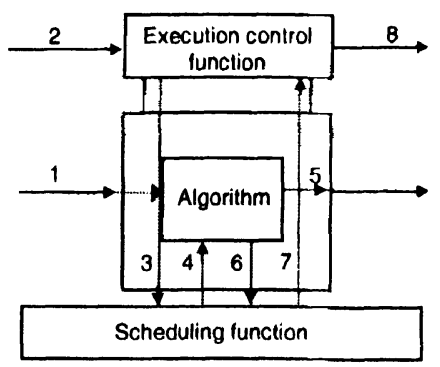

Figure 3 - Execution Model of an IEC 61499 Basic Function Block

Table 1 - Times according to IEC 61499

\begin{tabular}{|c|l|}
\hline Time Nr. & \multicolumn{1}{c|}{ Description of action } \\
\hline 1 & $\begin{array}{l}\text { Relevant input variables, coming from other FBs, are stable at } \\
\text { the input }\end{array}$ \\
\hline 2 & The event occurs at an event input \\
\hline 3 & $\begin{array}{l}\text { The FB execution control function signals the resource } \\
\text { scheduling function to schedule an algorithm for execution }\end{array}$ \\
\hline 4 & The scheduling function starts the algorithm \\
\hline 5 & $\begin{array}{l}\text { The algorithm process input values, in some cases, also } \\
\text { processes internally stored values and updates the output values } \\
\text { that are written to the function block's outputs }\end{array}$ \\
\hline
\end{tabular}




\begin{tabular}{|l|l|}
\hline 6 & $\begin{array}{l}\text { The algorithm execution has finished and notification of the } \\
\text { scheduling function to indicate that output data is stable at the } \\
\text { output }\end{array}$ \\
\hline 7 & The scheduling function invokes the execution control function \\
\hline 8 & $\begin{array}{l}\text { The execution control function signals an event at the event } \\
\text { output }\end{array}$ \\
\hline
\end{tabular}

The IEC 61499 execution model implies that each FB has to have a scheduling function. This scheduling function refers to one FB and is to guarantee that each time occurs in the correct order, at the correct priority (Lewis, 2000) and to sample the right data with its associated event determined through the WITH qualifier. Considering one FB or a network of interacting FBs in one resource there has to be a mechanism to detect and handle event inputs that occur faster than the necessitated time between time slot 1 and 8 . Additionally, the mechanism has to ensure that the resources' data of the network of FBs are consistent within the resource as well as to determine priorities and error handling for possible overloading of events in order to provide predictable IEC 61499 applications

Gaps of IEC 61499: Currently the IEC 61499 does not give detailed information what to do with overloaded input-events and its associated data and furthermore, it does not describe the characteristics and behavior of the scheduling function. It also does not include models for queuing input- or output events and its associated data. However, the standard points out these critical issues and mentions that "resources may need to schedule the execution of algorithms in a multitasking manner", but how to solve the existing problems is referred to be "implementation-dependent" (Part 1, 2000).

\section{CONCEPTS FOR IEC 61499 EXECUTION MODEL BEYOND THE STANDARD}

In this chapter we will introduce approaches for execution models that can be implemented on embedded execution platforms (slow processors, little memory and small or no operating system) in order to realize small and smart field devices. We also discuss the characteristics and behavior of the approaches to fill the gaps of the IEC 61499 standard (see 2.2).

All presented concepts shall be shown and compared via a little example application (Figure 4a) consisting of three Service Interface Function Blocks (SIFBs) and two Basic Function Blocks (BFBs). All of these FBs are located in one resource. The two SIFBs on the left generate events with their associated data (further referred as Input- SIFBs) that is processed by the two BFBs and finally published by the SIFB on the right (further referred as Output- SIFB). Each of these blocks is triggered by events. In case of the two Input- SIFBs this is any external event like a change of state of an external sensor. All other FBs are triggered by an event that is produced by another FB. So each FB has to be able to react on an event and autonomously start execution. If a multi- tasking system is used as suggested in the standard (see chapter 2) it seems to be obvious, at least in a first approach, to assign one task (- which may consist of one ore more threads) to each FB to allow parallel execution of all FBs containing one ore more internal algorithms. But this 
would definitely be too resource intensive to apply this concept on the embedded platforms we will introduce in section 4 below. So other solutions have to be found being able to run on these platforms. To overcome this problem two different approaches are discussed in the following subsections. In subsection 3.1 a single tasking and multi-threading concept with a definite reduction of the number of threads is presented, while the concepts in subsection 3.2 are based on conventional interrupt handling that can be found on small micro controllers.
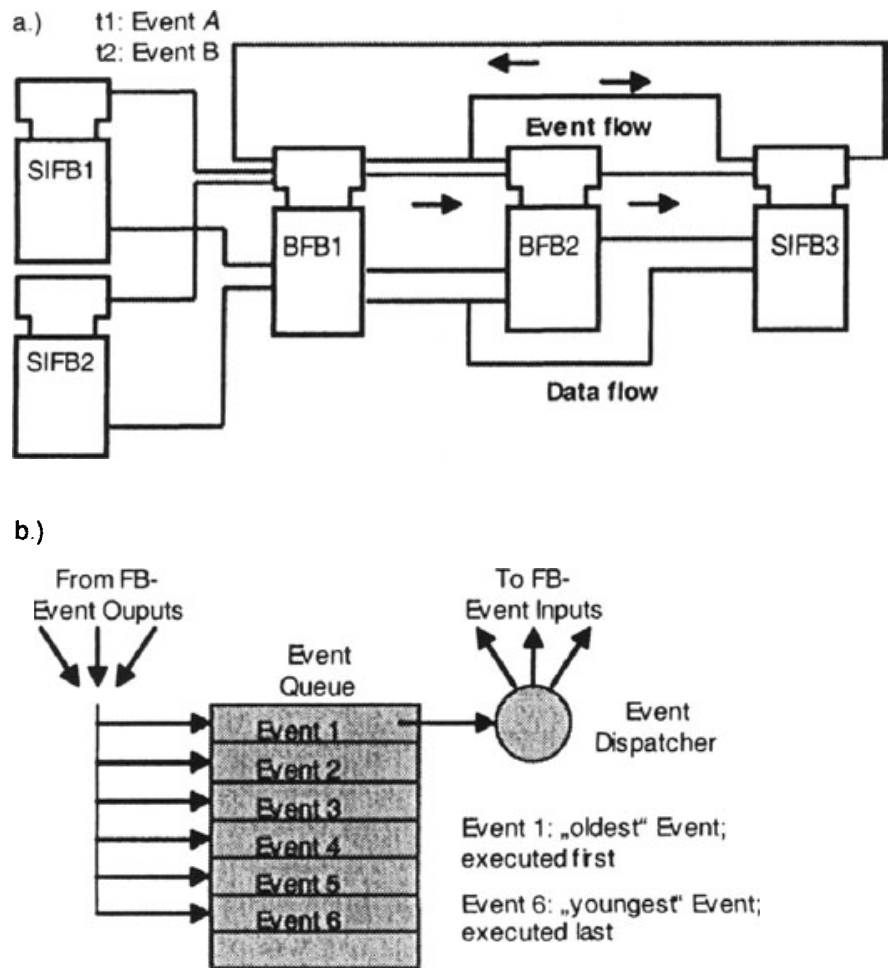

Figure 4 - Example a.) Application b.) Event Scheduler

\subsection{Execution Model Single Tasking with Multi-Threading}

The presented execution model of this chapter implies that the illustrated application (see Figure 4a.) runs in a single task containing several threads. Only one thread is to provide management services (Part 1,2000) within one resource (e.g.: building up the application in the resources) and one thread is assigned to each Input- SIFB, to allow the IEC 61499 application to react to external events. After the application is built up the threads of the Input- SIFBs are initiated and can generate output events in two ways, either by an external interrupt or the thread itself polls for certain service it should provide (e.g.: 8 digital inputs of an $\mathrm{V} / \mathrm{O}$ card). The following input- events of the BFBs are triggered by the generated output events of the InputSIFBs based on event function calls in a nested manner.

With this concept absolute parallel execution of the BFBs and Output- SIFB is only possible if different Input- SIFBs would initiate their execution (and so trigger different threads) and these SIFBs would be a part of separate, independent FBnetworks, latter because of the nested event function calls. If event outputs of 
different Input- SIFB are connected to the same FB (Figure 4a.) parallel execution is restricted caused by implemented threading mechanism based on the Java or C++ technology. To avoid data inconsistencies a synchronisation mechanism is implemented. This mechanism blocks an event coming from another thread to enter a FB while one thread is executing this block or succeeding blocks. The two restrictions to the parallel execution of FBs are:

- An execution triggered by an external event can only be done as long as the thread does not attempt to enter any FB that is blocked by another thread

- If a second input- event occurs at the same FBs while an initiated execution of a previous event has not finished this second event is ignored

The biggest drawback of this concept is that systems that support multi- threading are time and space consuming (see section 4.1 Java based systems). The benefit is that no central instance like a queue or scheduler is needed which reduces the complexity of implementing an IEC 61499 "like" execution model.

\subsection{Execution Models with Event/Data Scheduler}

In contrast to subsection 3.1 the following approaches are not based on multitasking or multi-threading operating system. Nonetheless, a method that allows an execution of FBs is achieved by scheduling events and data that are passed between FBs. Interrupt routines are used to trigger Input- SIFBs when a change of data is recognised at their inputs. All other FBs are executed according to different scheduler strategies described in the following subsections.

\subsubsection{Scheduling of Events only}

As illustrated in Figure 4b, all FB output events are set into a global event queue. The order of the events in the queue is equivalent to their order of occurrence. For example, event 1 shown in Figure 4b occurred first and so it was the first that was set into the queue. The dispatcher that knows the interconnection of the events takes event 1 first to trigger the corresponding FB. When the execution of this FB has terminated the dispatcher takes the next event from the queue and so on. A SIFBs' execution is triggered on external behalf at their service interfaces and not by any FB in the network. Due to that a SIFB cannot be triggered with $t$

he dispatcher. Instead interrupt routines are used for this purpose as mentioned above.

The concept has the drawback that events and corresponding data might be inconsistent. Consider the example shown in Figure 4a, The SIFB 1 generates at $t 1$ an event $A$ at its event output to communicate the receiving of new input data. After a period of time it sends at $\mathrm{t} 2$ an event $B$ at the same event output because input data changed again. Between the occurrence of event $A$ and $B, B F B 1$ was never executed because other function blocks were executed during that time. If after another period of time the dispatcher is able to take event $A$ from the queue to trigger BFB1, the FBs' input data corresponds to event $B$ and not to event $A$. Inconsistencies of data and events are the consequence, which may lead to undefined conditions in applications. 


\subsubsection{Scheduling of Events and Data}

By storing not only events in a queue but also the corresponding data, it is guaranteed that when the dispatcher starts the execution of a definite FB by triggering it with an event the corresponding data is present at the data inputs of the block. To store also data in the global queue instead of just events (3.2.1) requires a lot of memory, time-consuming data copying and performance. If execution is too slow the dispatcher is not able to take as many events from the queue, as are put into the queue in the same period of time. This leads to unacceptable delays or even to an overload of the queue.

\subsubsection{Definite Ignoring of Events}

The standard defines that "multiple occurrences of an event at the same event input may be lost ... while waiting for ECC to finish ... The detection and processing of such loss is an implementation- dependent feature." (Part 1, 2000)

This is equivalent with the fact that an event may be ignored if the execution of the event's predecessor at the same event input of the FB is not finished when this new event occurs. This allows adopting the concept of event queues. Events are only put into the queue if no event of the same event output is already in the queue waiting to be executed. Otherwise the event is deleted. This leads to a shorter queue and so to the fact that waiting time of accepted events for getting executed is reduced.

Nonetheless it is important to provide mechanisms to detect event losses, as the number of lost events is a characteristic value for the user to estimate the systems workload. Another benefit of definite ignoring of events is that the problem of data inconsistencies like it occurred in 3.2.1 is significantly reduced. If all data inputs at a FB are assigned to their corresponding event by the WITH construct (see section 2), present data at the FBs data inputs may be used for execution; no matter if the ECC is executed immediately after the event occurred or if the execution is delayed because "older" events in the queue are executed first. Summarized advantages of this concept:

- Short event queue and so reduced waiting time for events to get executed

- Inconstancies of data and events can be avoided by proper use of the WITH construct

- No multi-tasking concept is required as SIFBs are handled with interrupts while all other FBs are executed sequentially 


\subsubsection{Hybrid Form}

Due to the mentioned advantages the concept presented in 3.2 .3 is most suitable for the aspired low performance execution platforms. Nonetheless one has to be aware that the ignoring of events like it is done in approach 3.2.3 is not acceptable for all applications. Consider a counter- FB whose task is to count its input events. In this case events may not be ignored even if the processing of the preceding event is not finished yet.

This can be solved by a hybrid form that is based on the concept of definite ignoring of events (3.3.3) with the extension of special event- and data- queuing for critical FBs.: Critical FBs whose input events may never be ignored have to be treated differently by the event queue. Every event that is calling such a special FB has to be put into the queue, no matter if a predecessor of this event is in the queue already or not. So it is guaranteed that all occurred events are handed on to the FB. As multiple occurrences of events are allowed for critical FBs additional data queuing for these FBs is required to avoid data inconstancies.

As a conclusion of this the hybrid form requires priorities of events with its associated data, which have to be set by the user. Therefore the user has to analyse his application before implementing it to be sure that the application is predictable.

\section{CURRENT STATE OF WORK}

Profactor and the Automation Control Institute are working intensively on the evaluation of the different execution models by means of various platforms. At the moment our work focuses on Class 1 devices (IEC, 2000). This chapter gives an overview of the platforms and describes their kernel (Java based or $\mathrm{C} / \mathrm{C}++$ based), interfaces and memory resources with respect to the execution model and problems.

\subsection{Java-Based Execution Platforms}

The Java technology for embedded systems attracts interest all over the world, due to its advantages (Sun, 2002). Therefore we introduce two small Java ${ }^{\mathrm{TM}}$ based execution platforms for the possible use of smart field devices.

\subsubsection{TINI}

TINI stands for Tiny Internet Interface and it is a small $\mathrm{Java}^{\mathrm{TM}}$ execution platform that is built up by one DS80C390 (a 8051 derivative) micro controller, one flash ROM, one static RAM, an Ethernet controller and a real-time clock. The micro controller itself is equipped with a CAN interface, 2 Serial ports, a 1-wire interface and several TTL IOs. The TINI runtime environment can be divided into two categories: native code execution directly by the micro controller and an API (Application Programming Interface) interpreted as byte codes by the TINI Java ${ }^{\mathrm{TM}}$ virtual machine (TINI, 2002) which size is less then 40 kilobytes. Applications can be executed in Java using the supported packages or if there exist stringent real-time requirements, native libraries can be loaded within an application.

The IEC 61499 runtime that allows the execution of IEC 61499 applications was implemented with the Java technology (runtime is currently available on 
http://www.holobloc.com). The TINI operating system provides facilities for multitasking and multithreading. Due to these features, each FB type is represented as a Java class with defined interfaces and the execution model is based on a multithreading methodology described in section 3.1 above. For verification purposes, we extended the TINI with starter kit boards providing a parallel interface with up to 24 discrete $\mathrm{I} / \mathrm{O}$ points. Successful tests were done on a small assembly test bed..

Shortcomings: The bottleneck of the TINI platform is its memory that is limited to $512 \mathrm{kByte}$ Flash ROM and optional $512 \mathrm{kByte}$ or 1 Mbyte SRAM and last but not least its slow performance. The Flash ROM is already used by the TINI OS, the TINI JVM, class libraries and command interpreter (TINI, 2002). The file system, the IEC 61499 runtime and the IEC 61499 FBs are stored in the non-volatile SRAM. The TINI board represents a device Class 1 (IEC, 2000). By downloading a programmed application from an IEC 61499 Editor, the application will be built up on the TINI's SRAM. This process is very resource intensive because of instancing the runtime's classes and the application's FB classes with all its interconnections to provide a runnable application. Additionally, the event-triggered methodology is based on Java synchronized function calls in a nested manner. This fact causes several problems with memory overflow and determinism if the system engineer doesn't consider these nested event function calls.

\subsubsection{SaJe}

SaJe stands for Systronix aJile Euroboard that is equipped with an aJ100 native $\mathrm{Java}^{\mathrm{TM}}$ processor $(100 \mathrm{MHz})$ from aJile systems, 1MB SRAM, 4MB FLASH and several communication interfaces including, among other things 2 Serial Ports, Ethernet, 1-Wire and expansion board socket. The J2ME CLDC (Sun, 2002) serves as runtime environment of the SaJe. The IEC 61499 runtime and the different FB types are implemented as on the TINI chip. For verification purposes we extended the board with a parallel interface with up to 24 IOs. By means of this interface we could also control a small assembly test bed.

Shortcomings: There are two main advantages compared to the TINI platform, namely the better execution performance and the size of the memory. Due to the 4MB of Flash ROM, there is the possibility to store the classes of both the runtime and the FB into the Flash in order to build up larger applications in the SRAM. Therefore the disadvantages regarding nested event function calls and the threading model are the same.

\subsection{C++ Based Execution Platforms}

The presented Java platforms are not capable of the defined requirements for small, distributed control platforms. Other approaches are necessary to reach realtime execution with low performance platforms. For instance, $\mathrm{C} / \mathrm{C}++$ implementations are less memory and time consuming than applications written in Java. Furthermore the number of embedded Java platforms accepted by automation industries is restricted. For these reasons an implementation in $\mathrm{C}++$ was developed. To use existing know-how the implementation was based on the work done in Java. 
Current state: Due to the wide distribution the $\mathrm{C} 166 \mu$-controller from Infineon was chosen for a first implementation. Each FB is represented as a class in C++. These classes are compiled and linked into one executable file for the specific system (e.g. Intel hex file) that is stored in the flash. The application is built on instances of these classes in the RAM. The IEC 61499 execution model is implemented respectively to the methodology of Definite Ignoring of Events (3.2.3). SIFBs are triggered via hardware interrupts of the C166 $\mu$-controller. For the communication between the devices communication SIFBs have been implemented using CAN .

Additionally the $\mathrm{C}++$ execution platform has been compiled for the IPC@CHIP from Beck. On this system communication function blocks for Ethernet are available.

\section{CONCLUSIONS AND FUTURE WORK}

As described in chapter 3, a hybrid form of the introduced execution models may fulfill requirements like determinism, data consistence and event losses within one resource. Therefore events with their associated data may be classified according to the demands of the application (e.g.: a closed-loop control may need the latest event and the latest data, in contrast to that a counter may need all events with all associated data). System- engineers have to be aware of this classification while programming an IEC 61499 application.

Nonetheless, our test cases showed that with the presented concepts of the extended IEC 61499 execution models it is possible to run IEC 61499 applications on low-cost embedded systems in order to control several test beds, large and small sized ones.

In the near future our team will extend the variety of IEC 61499 embedded platforms and evaluate each of them by applying different execution models and test them with several test beds.

\section{REFERENCES}

1. Christensen J.H. "IEC 61499: An Open Architecture for Scalable Flexible Automation", http://www.holobloc.com/papers/sfarch2.zip

2. HMS European Module BPR-CT-97-9000 Deliverable D1.1-1/D1.1-1 Holonic System Architecture; University of Keele, 1998, Keele

3. Holobloc Homepage, http://www.holobloc.com

4. Lewis R.W. Modeling control systems using IEC 61499, ISBN: 0852967969

5. IEC (International Electrotechnical Commission): PAS 61499-1, "Function Blocks, Part 1 Architecture". Geneva, 2000.

6. TINI software, http://www.ibutton.com/TINI/software/index.html

7. SaJe "Real Time Native Execution", http://www.systronix.com/saje/index.htm

8. "Embedded Systems go Real Time with Java ${ }^{\text {TM }}$ Technology", http://java.sun.com/features/2001/04/embed.html 